\title{
Space Lidar and Space Optics
}

\author{
Andreas Fix ${ }^{1} \cdot$ Giampiero Naletto $^{2} \cdot$ lan Hutchinson ${ }^{3} \cdot$ Nikos Karafolas $^{4} \cdot$ Wolfgang Riede $^{5} \cdot$ Arnaud Heliere $^{4}$. \\ Bob Menzies $^{6} \cdot$ Haris Riris $^{7}$
}

Published online: 13 November 2019

(c) CEAS 2019

This is the second Special Issue on Space Optics of the CEAS Space Journal, 2 years after the first. As in the first Special Issue, the collected papers are largely based on contributions presented at the last International Conference on Space Optics-ICSO-held in October 2018 in Chania, Greece. ICSO is the largest multidisciplinary meeting of experts working in all disciplines of optical, optoelectronic and photonic technologies for space applications and held every 2 years; it is a pre-eminent event for reporting about advances in the field especially in Europe, and is attended every year by an increasing number of scientists and technologists.

The special issue in hand is separated into two parts: "Space Lidar" and "Space Optics".

Lidar, light detection and ranging, using lasers is an active remote sensing technique that has become an important backbone for atmospheric and land surface remote sensing. Currently, we anticipate a golden age for lidar in space. Today's lidar systems have reached a high degree of maturity and sophistication that increasingly allows for their successful implementation in space missions.

Since the first Special Issue on Space Optics of the CEAS Space Journal in 2017, as many as four lidar missions were launched into space:

Nikos Karafolas

Nikos.Karafolas@esa.int

1 DLR Institute of Atmospheric Physics, Oberpfaffenhofen, Germany

2 Department of Physics and Astronomy, University of Padova, Padova, Italy

3 Department of Physics and Astronomy, University of Leicester, Leicester, UK

4 European Space Agency/ESTEC, Noordwijk, The Netherlands

5 DLR Institute of Technical Physics, Stuttgart, Germany

6 NASA Jet Propulsion Laboratory, Pasadena, CA, USA

7 NASA Goddard Space Flight Centre, Greenbelt, MD, USA
- Aeolus, the first European lidar mission and the first spaceborne lidar to measure global winds, was launched by ESA on 22 August, 2018.

- The Ice, Cloud, and Land Elevation Satellite-2 (ICESat-2) was launched on September 15, 2018, and will measure changes in the surface topography of the Earth, with main emphasis on the cryosphere.

- Since 20 October, 2018, the European Space Agency's and JAXA's BepiColombo mission is on its 7-year flight to Mercury to measure the topography, surface roughness, tidal deformations and other geodetic properties of this planet.

- Finally, the GEDI (Global Ecosystem Dynamics Investigation) ecosystem lidar payload was launched on 5 December, 2018. This lidar targets the determination of the 3-d structure of forests and is operated by NASA on board of the International Space Station (ISS).

In view of such remarkable developments, the editorial board on Optics, Optoelectronics and Photonics of the CEAS Space Journal decided to dedicate the first part of this special issue to "Space Lidar" and related developments.

The first paper in this volume describes a satellite laser ranging (SLR) application. Here, a satellite ground station was established using a laser with a repetition frequency of as high as $100 \mathrm{kHz}$. The challenges and benefits of using such very high repetition rates for SLR are examined in theory and also in practice. This approach demonstrates benefits in terms of the pulse energy transmitted and reduced statistical error and may lead to more cost-effective SLR systems.

The following two papers also relate to laser ranging, and give the details of two planetary laser altimetry missions. In the first one, the main elements of the BepiColombo Laser Altimeter experiment, BELA, are described. The BepiColombo mission was launched on 20 October, 2018 , and BELA will provide altimetry measurements of Mercury. The paper provides an update on the main elements of the instrument, discusses problems solved during instrument development and the results from the ground testing. 
The second paper outlines the Ganymede Laser Altimeter (GALA), which is intended to obtain, as part of the Jupiter Icy Moons Explorer (JUICE) mission, topography data from icy Jupiter moons by the end of the 2020s. Currently, the mission is in its implementation phase and this paper reports on the objectives, instrument design and instrument performance.

Following this section, three papers describe important components for satellite ranging and spaceborne lidar applications. In the first paper, the design and qualification of a retroreflector for CubeSats is described that is expected to provide an effective means for coarse attitude control for satellites. The next paper reports on the implementation of a microchannel plate photomultiplier tube. Important performance parameters such as quantum efficiency, spectral response, timing behavior and nonlinearities are discussed in the context of potential lidar applications. Tunable laser systems are vital for lidar applications such as resonance fluorescence or differential absorption lidar. The final paper in this section reports in this context on the development of a diode pumped chromium-doped alexandrite laser. The authors report on the design and performance evaluation of such device, which shows considerable promise for future spaceborne resonance lidar missions for mesospheric studies.

Going further, into the direction of atmospheric applications of spaceborne lidar, the next contribution then reports on the development steps, integration, and extensive testing efforts of The ATmospheric LIDar, ATLID, which is part of the payload of the Earth Cloud, Aerosol and Radiation Explorer (EarthCARE) mission jointly conducted by ESA and JAXA. While ATLID will observe the vertically resolved profiles of aerosol and clouds from 2022 onward, the French-German climate mission MERLIN (Methane Remote Sensing LIdar Mission), scheduled for launch in 2024, has as its goal the measurement of the vertical column of the second most important anthropogenic greenhouse gas, methane. In this context, the subsequent paper gives an overview on the payload design of the MERLIN lidar, performance calculations and reports on details concerning the present status of subsystem development. This includes the Nd:YAG laser pumped optical parametric oscillator as the tunable lidar transmitter, the receiving system, calibration chain, control units or thermal control hardware. One particularly critical submodule of MERLIN is the frequency reference unit, as the knowledge accuracy on the absolute wavelength of the outgoing laser beam is a prerequisite for accurate monitoring of methane. The design of this module and the obtained frequency stability and accuracy are discussed in detail in the next paper. The last paper in this section also relates to the measurement of methane. Here the authors describe in detail the technological challenges of measuring methane from space with a lidar, and discuss spectroscopic issues, and suitable instrument approaches particularly focused on tunable laser transmitters for both spaceborne and airborne applications.

The contributions in the "Optics" section are spread over a large number of different themes, demonstrating the large range of applications covered and the relevance or prominence of optical technologies in both Earth and Space Science. We start with the characterization of instruments or devices on board of ESA space missions, already in flight or soon to be launched. Then a couple of papers describe new instrument concepts and designs, mainly dedicated to remote sensing observations. These papers capture perfectly the key features of the next generation of space instruments, high compactness and reliability. After the initial papers dedicated to the instrument description, the three following papers feature a new topic for space, that is, the use of active/adaptive optics. This topic is becoming increasingly relevant to meet the demand for improved performance from space assets. Finally, the last papers are a collection of the most recent works on specific technologies fundamental for the development of new and more performing devices to be used on satellites.

The first paper of the Optics section describes the radiometric calibration of the stereoscopic camera on board of BepiColombo, the ESA mission launched last year to reach Mercury. Instrument calibration is always a very critical activity for getting reliable information from a remote sensing instrument. As usual in space astronomical instrumentation, where each instrument is a prototype, a specific on-purpose calibration activity had to be performed, with a dedicated facility and with the implementation of dedicated analyses. This paper well describes the work to be done to characterize CMOS sensors for astronomical space observations, showing significant differences with respect to the calibration of the much more consolidated CCD sensors typically used in space. The second paper belonging to this section covers another ESA mission, Solar Orbiter, that will be launched by February 2020. It will be the first space mission to provide a view of the Sun poles, and will orbit around the Sun at perihelion distances within the Mercury orbit, making it one of the hottest environments ever sustained by satellite optical payload. One of the instruments onboard is the Polarimetric and Helioseismic Imager ( $\mathrm{SO} /$ PHI) that will provide maps of the magnetic vector and of the line-of-sight velocities in the solar photosphere. SO/PHI is equipped with two telescopes and a common camera, and the very high precision Feed-Select Mechanism realizes the selection between the two telescopes. This paper describes this mechanism, its design features, and its performance verification and qualification. This mechanism not only had to satisfy very stringent requirements because of the need of accurate optical alignment, but had also to include a fail-safe system being an instrument single-point failure. 
Concerning the new instrumental concepts, the third paper of this Section describes NanoCarb, an innovative compact Fourier-transform imaging spectrometer dedicated to the measurement of $\mathrm{CO}_{2}$ and $\mathrm{CH}_{4}$. This instrument satisfies the big challenge of realizing a very accurate spectrometer for remote sensing observation in the small volume allowed by a $50-\mathrm{kg}$ class satellite. This is a very innovative instrument, designed having in mind its application to a constellation of small and low-cost satellites. The described performance of this instrument is very promising, and shows that it is possible to reach extremely challenging results with present technologies. The development of this instrument is really advanced, and an experimental proof of concept is planned in the near future in the framework of the Horizon 2020 project Space CARBon Observatory (SCARBO). The subject of the fourth paper of this Section is very similar: in this case, the new concept of a compact spatial heterodyne interferometer for the observation of faint emissions in the atmosphere. The example implementation of this payload as a middle atmosphere temperature sounder is described. This type of instruments is particularly suited for the deployment on nano- or micro-satellite constellations. Together with the preceding paper, this paper demonstrates the large effort devoted by scientists, technologists and space companies to the realization of more compact instruments, and the development of large networks of small and cheap satellites.

The following three papers are dedicated to a topic that has been largely studied for ground telescopes but not yet for space applications, that is, the use of active and adaptive optics to minimize dynamical optical aberrations. At present, the large majority of space instruments does not suffer from this problem. However, Earth observation from satellites is affected by atmospheric fluctuations. Future optical communication, with more stringent requirements on the pointing capabilities, or the auxiliary system dedicated to the control of satellite flight formations, would definitely benefit from an optical system, which could adapt to a dynamic situation. Another very important reason to develop this technology for space applications is the need of having larger and larger telescopes, which have to be light, segmented and stowable or potentially assembled in space. The co-alignment of the segments has to be performed remotely and periodically checked during the mission lifetime, and this could be done only by means of active optical systems (for example, the James Webb Space Telescope 6.5-m-diameter segmented primary mirror will be actively controlled during its science operations). Thus, given the importance of this new space technology, we are very pleased to have some dedicated papers in this Special Issue. The first paper on this topic deals with the manufacturing of mm-thick shells of glass suitable for the realization of active optics for future space telescopes. In this paper, a novel technique is described in which thin flat shells of glass are shaped by replication via hot slumping followed by computer-controlled polishing or ion beam figuring for providing the required optical quality. The in-flight deformations induced on these thin glass shells will then be compensated for by a discrete set of adjustable actuators on the mirror backside. The second paper demonstrates how it is possible to increase the safety of unimorph piezoelectric mirror during the critical launch phase. These special mirrors consist of a single-crystal $\mathrm{Si}$ wafer $0.5 \mathrm{~mm}$ thick to be used as active optics, and it is fundamental to be able to guarantee their integrity during the extreme conditions of the launch. The proposed method has been validated by a dedicated experiment, and it is intended to be used in future ESA space missions. The third paper on this topic theoretically treats the significant problem of estimating deformation and misalignment of a three-mirror telescope by means of the minimum mean square error estimation method. Using this method, the aberrations in the field of view of the telescope are minimized with just a few wave front measurements in the focal plane and consequent mirror surface error compensation. A comparison with the more classical least square approach shows that this method is more reliable and accurate.

Concerning the papers dedicated to more specific technologies, we start with another very strategic topic, that is, the capability of bringing quantum experiments to space. Despite the increasing efforts, many difficulties are still left to be overcome. In particular, the means to produce and handle clouds of atoms is extremely challenging and at the limit of present technologies. Here the development and qualification of miniaturized, ultra-stable optical setups for optical cooling and trapping of cold atomic gases on atom chips are described, showing that we are getting closer to bringing quantum technologies to space.

Then there are two papers on reflective coatings for visible and infrared ranges: even if this topic is no longer considered fashionable, a lot of research is still conducted to optimize the mirror performance in these spectral ranges. For example, a critical element for space optics is the optimization of antireflection coatings to eliminate the source of many undesired features on the focal plane such as spurious reflections and ghost images. In the first paper on this topic, we can find the results obtained by applying thin film technology for the production of normal incidence broadband antireflection coatings both for the visible 400-1100 nm spectral range and for the mid-IR $1.5-15 \mu \mathrm{m}$ range. Very important in this paper are the results obtained in particular conditions rather common in optical applications, such as the cases of $45^{\circ}$ oblique incidence, and illumination with beam apertures as large as $\pm 50^{\circ}$. Another very interesting topic in this field is how to protect delicate reflecting coatings under harsh environmental conditions. In the following paper of this Section we can find the performance of optimal protecting layers for silver-coated reflectors, very 
often used for both space and ground applications, showing that different materials should be selected as a function of the working environment of these mirrors. In particular, it is shown that silicon nitrite overcoating should be preferred under difficult environmental conditions with respect to the more traditional $\mathrm{Al}_{2} \mathrm{O}_{3}$ and $\mathrm{SiO}_{2}$ coatings.

The following paper covers another critical optical technology for space, free space to fiber coupling of optical signals. Fiber coupling, in fact, is becoming an increasingly common challenge in many space applications, for example, for lidar, optical communications, laser ranging, and quantum sensors. Often the very stringent requirements in alignment and stability are challenging to reach. Here, a novel optical beam steering technique for fiber to free-space to fiber coupling is presented, simply based on a glass plate and a pair of glass wedges. The technique proposed in this paper makes it possible in a rather simple and convenient way to adjust the angle and position of a beam with extreme precision maintaining at the same time a very high degree of stability in a rather compact design.

The last two papers are dedicated to an important small device used in space systems for photon and particle detection, the microchannel plate (MCP). Both these papers deal with the use of MCPs on board of the upcoming ESA mission JUICE to Jupiter. In the first paper, there is the thermal characterization of the MCP detector for the Jovian Energetic Neutrals and Ions (JENI) experiment, dedicated to the detection of energetic neutrals and ions. This paper describes how the cold environment of this deep-space mission will impact the performance of MCPs, changing their intrinsic resistivity and consequently their gain. The knowledge of how the MCP resistivity changes with temperature is fundamental for the instrument performance, because it will define how to tune the high voltage applied to the MCP to maintain the nominal performance of the JENI sensor. The second paper describes the behavior of MCPs in the extreme Jovian environment, where many charged particles are trapped by
Jupiter's intense magnetic field. The radiation-induced background noise provided by the environment can potentially contaminate the science signal; this is definitely one of the main challenges of this mission, together with the radiation hardness of the electronics. Here the changes of MCP performance after strong irradiation are described, providing some important information about the requirements to be satisfied by the protecting shield to maintain the required signal to noise ratio from these devices.

We believe that the wealth of papers presented in this Special Issue reflects the amazing developments in the field of Space Optics and, in this particular period of time, the emergence of active optical instruments in the form of lidar offers a powerful new engineering tool to be exploited by the scientific community in the years to come.

The Editors would like to thank all the authors for providing such detailed manuscripts of their advanced activities as well as the reviewers who provided excellent inputs that contributed to the improvement of the submitted manuscripts.

The CEAS Associate Editors on Optics, Optoelectronics and Photonics

Andreas Fix

Giampiero Naletto

Ian Hutchinson

Nikos Karafolas

Wolfgang Riede

The Guest Editors for the Special issue on "Space Lidar" Arnaud Heliere

Bob Menzies

Haris Riris

Publisher's Note Springer Nature remains neutral with regard to jurisdictional claims in published maps and institutional affiliations. 